\title{
Magnetic Doppler imaging of II Peg
}

\author{
Oleg Kochukhov ${ }^{1}$, N. Piskunov ${ }^{1}$, I. Ilyin ${ }^{2}$ and I. Tuominen ${ }^{3}$ \\ ${ }^{1}$ Department of Physics and Astronomy, Uppsala University, 75120 Uppsala, Sweden \\ ${ }^{2}$ Astrophysikalisches Institut Potsdam, An der Sternwarte 16, D-14482 Potsdam, Germany \\ ${ }^{3}$ University of Helsinki, PO Box 14, 00014 Helsinki, Finland
}

\begin{abstract}
Rotational modulation of the intensity and polarization spectra of magnetic stars offers a unique possibility to reconstruct the structure of surface magnetic fields and to investigate their relation to cool starspots. We have developed a new magnetic Doppler imaging code which aims at self-consistent temperature and magnetic mapping of cool active stars. Here we present magnetic Doppler imaging analysis of high-resolution circular polarization observations of the active star II Peg. We demonstrate that a self-consistent approach to magnetic inversion unveils stronger magnetic fields than found previously through disjoint analyses of polarization and intensity observations of active stars.
\end{abstract}

Keywords. Stars: activity - stars: atmospheres - stars: magnetic fields - stars: II Peg

\section{Introduction}

Rotational modulation of the intensity and polarization spectra of active stars offers a possibility to reconstruct 2-D maps of the photospheric magnetic fields and temperature spots, thus providing a key constraint for the theoretical modelling of the stellar activity and dynamo. Until now Zeeman Doppler imaging of the active late-type stars (Brown, Donati, Rees, et al. 1991; Donati 1999; Hussain, Donati, Cameron, et al. 2000) was based upon a non-simultaneous and simplified interpretation of the average (least-squares deconvolved) Stokes $I$ and Stokes $V$ spectra. In contrast, detailed and physically consistent methods of the stellar magnetic field mapping have been developed (Piskunov \& Kochukhov 2002; Kochukhov \& Piskunov 2002) and successfully applied (Kochukhov, Piskunov, Ilyin, et al. 2002; Kochukhov, Bagnulo, Wade, et al. 2004) to the high-resolution circular and linear polarization observations of the early-type magnetic stars.

Improvements in the observational techniques and instrumentation have recently made it possible to detect polarization signatures inside individual atomic (Petit 2006) and molecular (Berdyugina, Petit, Fluri, et al. 2006) lines of the late-type stars. With these observational data in mind, we have developed a new Magnetic Doppler imaging (MDI) code Invers13. Our magnetic inversion code reconstructs iteratively two-dimensional distributions of temperature and three components of the magnetic field vector using high-resolution spectropolarimetric time series recorded in the Stokes $I$ and $V$ or all four Stokes parameters. Inversions employ the Levenberg-Marquardt minimization procedure and are constrained with the Tikhonov regularization (Piskunov \& Kochukhov 2002).

The modelling of the magnetic field and cool spots is self-consistent, i.e. the local intensity and polarization profiles are computed taking into account the Zeeman effect and the presence of temperature inhomogeneities at the same time. This allows us to deduce correct field strength inside cool spots. 

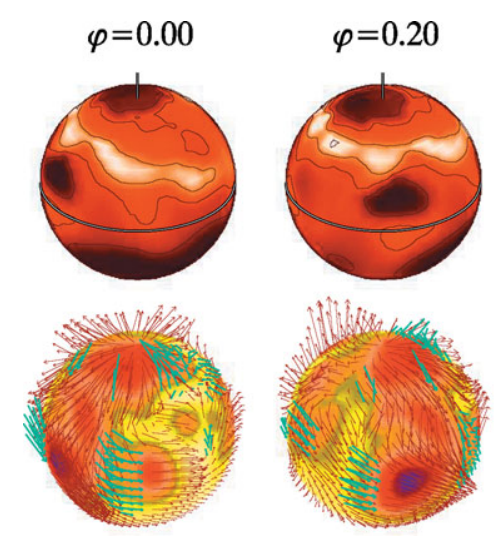
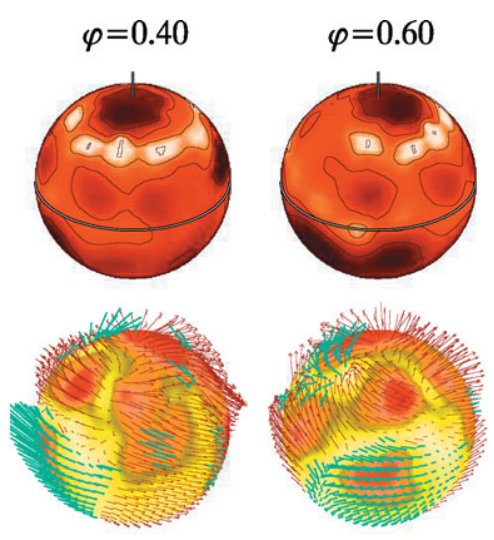

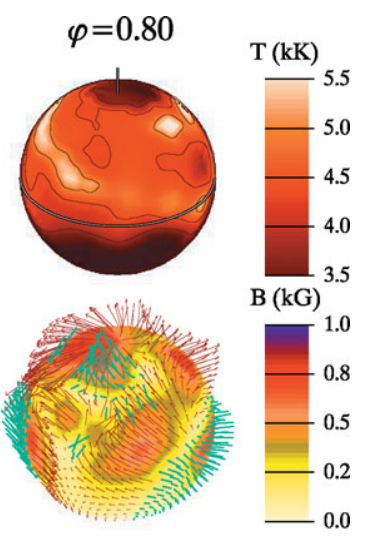

Figure 1. Temperature spot distribution (upper panel) and magnetic field geometry (lower panel) inferred from the magnetic inversion of the Stokes $I$ and $V$ spectra of II Peg.

\section{Magnetic field topology of II Peg}

We have applied the new MDI code to the circular polarization observations of II Peg obtained with the SOFIN spectrograph at the Nordic Optical Telescope (La Palma). High-resolution spectra in the Stokes $I$ and $V$ parameters were obtained at 8 rotational phases in June-August 2004. These observations are characterized by the resolving power $R=70000$ and have typical $S / N>400$. The spectra were reduced with the 4 A software package (Ilyin 2000).

Adopting the rotation period and atmospheric parameters of II Peg determined in previous model atmosphere and Doppler imaging studies of this star (Ottmann, Pfeiffer, Gehren 1998; Berdyugina, Jankov, Ilyin, et al. 1998), we have carried out magnetic inversion simultaneously with the reconstruction of temperature map using profiles of the three magnetically sensitive Fe I lines at $\lambda \lambda$ 5495-5507 $\AA$ and two strong Ca I lines 6102.7 and $6122.2 \AA$. Resulting magnetic and temperature distributions are presented in Fig. 1. We find that at the surface of II Peg the field strength reaches up to one kG. Some strong-field regions coincide with low-temperature regions. The magnetic field inferred in our analysis is stronger than typically found in previous ZDI mapping of cool active stars based on the LSD technique and weak field approximation (e.g., Donati 1999).

\section{References}

Berdyugina, S. V., Jankov, S., Ilyin, I., Tuominen, I., \& Fekel, F. C. 1998, A\&A 334, 863

Berdyugina, S. V., Petit, P., Fluri, D. M., Afram, N., \& Arnaud, J. 2006, in Astronomical Society of the Pacific Conference Series, eds. R. Casini, \& B. W. Lites, ASP Conf. Ser. 358, 381

Brown, S. F., Donati, J.-F., Rees, D. E., \& Semel, M. 1991, A\&A 250, 463

Donati, J.-F. 1999, MNRAS 302, 457

Hussain, G. A. J., Donati, J.-F., Cameron, A. C., \& Barnes, J. R. 2000, MNRAS 318, 961

Ilyin, I. V. 2000, Ph.D. thesis, University of Oulu Finland

Kochukhov, O. \& Piskunov, N. 2002, A\&A 388, 868

Kochukhov, O., Piskunov, N., Ilyin, I., Ilyina, S., \& Tuominen, I. 2002, A $\mathscr{J} A$ 389, 420

Kochukhov, O., Bagnulo, S., Wade, G. A., Sangalli, L., Piskunov, N., Landstreet, J. D., Petit, P., Sigut, T. A. A. 2004, A\&A 414, 613

Ottmann, R., Pfeiffer, M. J., \& Gehren, T. 1998, A\& A 338, 661

Petit, P. 2006, in Astronomical Society of the Pacific Conference Series, eds. R. Casini, and B. W. Lites, ASP Conf. Ser. 358, 335

Piskunov, N. \& Kochukhov, O. 2002, A\&A 381, 736 